\title{
Confined systems within arbitrary enclosed surfaces
}

\author{
B L Burrows ${ }^{1}$, and M.Cohen ${ }^{2}$ \\ ${ }^{1}$ Mathematics Section \\ Faculty of Computing, Engineering and Sciences \\ Staffordshire University, College Road, Stoke-on-Trent,ST4 2DE UK \\ e-mail:B.L.Burrows@staffs.ac.uk \\ ${ }^{2}$ Department of Physical Chemistry \\ The Hebrew University of Jerusalem \\ Jerusalem 91904, Israel \\ e-mail:maurice@fh.huji.ac.il
}

A new model of electronic confinement in atoms and molecules is presented. This is based on the electronic flux $\mathrm{J}$ which is assumed to vanish on some notional bounding surface of arbitrary shape. $\mathrm{J}$ is necessarily calculated using an approximate wave-function, whose parameters are chosen to satisfy the required surface conditions. This model embraces the results of all previous calculations for which the wave-functions or their derivatives vanish on conveniently-shaped surfaces, but now extends the theory to more general surfaces. Examples include one-centre hydrogen-like atoms, the valence state of $L i$ and the two centre molecular systems $\mathrm{H}_{2}^{+}$and $\mathrm{HeH}^{++}$.

Keywords: Atomic and Molecular Confinement, Electronic Flux, Valence States. 


\section{Introduction}

Much of the recent work on solutions of the non-relativistic Schrödinger equation has focussed on confined atomic and molecular systems. This stems from the realisation that physical systems are not of infinite extent. A representative set of papers can be found in [1] and an additional selection in references [2-10] .

We begin by recalling that almost all previous treatments have specified neither the nature or the source of confinement, but have simply assumed that the state function $\psi$ vanishes everywhere on the confining surface. A more realistic model can be suggested; this is based on the electronic charge flux $J$ over some arbitrary-shaped enclosing surface $\mathrm{S}$, and is defined by

$$
J=\int_{S} \frac{\partial \rho}{\partial n} d S, \quad \rho=\psi^{*} \psi=|\psi|^{2}
$$

where $\mathbf{n}$ denotes the unique outward unit normal at each point of the bounding surface.

Any valid approximate $\psi$ will naturally be a function of a set of embedded parameters a,b,c...:

$$
\psi=\psi\left(a, b, c_{. .} \mid x, y, z . .\right)
$$

making $J$ a function of the same set of parameters. We now propose to choose these so that $J=0$.

In the illustrative calculations presented here we may choose $\psi$ real and we note that in the particular case of a $\mathrm{H}$-like atom inside a spherical box of radius $\mathrm{R}$, there are two elementary solutions:

1. $\psi=0$ everywhere on $S$, and

2. $\frac{\partial \psi}{\partial n}=0$ everywhere on $\mathrm{S}$.

This follows since $\mathbf{n}=\hat{\mathbf{r}}$, the radial unit vector and the solution separates in the form $\psi=f(r) Y(\theta, \phi)$ so that

$$
J=2 f(R) \frac{d f(R)}{d r} \int_{0}^{2 \pi} \int_{0}^{\pi} Y^{2} R^{2} \sin (\theta) d \theta d \phi
$$

The integral is positive so that $J=0$ implies that $f=0$ or $\frac{d f}{d r}=0$ at $r=R$.

We have previously derived $[4,5]$ the (unnormalised) ground state radial solution of a H-like atom of nuclear charge $\mathrm{Z}$ :

$$
\psi=(2-Z r) \exp (-Z r / 2), \quad 0<r<R=\frac{2}{Z}
$$

which vanishes on the boundary $r=R$. Note however that it is not zero for larger values of $\mathrm{r}$ and forms an excited state for the unconfined system. For the confined system, it has usually been assumed that the traditional unconfined 
problem is modified by retaining the usual Coulomb potential $-Z / r$, only in the range $0<r<R$ and changing this in $r \geq R$ to a potential which is infinite in a bounding region so that the solution is everywhere zero on the surface. Essentially the model describes the effect of the confining potential, which is assumed to act in $r>R$, rather than describing the form of the potential beyond the bounding surface. An example of the second type of solution may be illustrated by considering the derivative of the wave function in Eq. (4) which is proportional to

$$
(4-Z r) \exp (-Z r / 2)
$$

so that it vanishes at $r=4 / Z$ and consequently we may regard $\psi$ of Eq. (4) as the wave function for a confined system in $0<r<R=4 / Z$. Note that since $\psi$ has a zero at $r=2 / Z$ then it is not the ground state solution confined in this interval. The external potential required to confine the atom in this way is different from the infinite potential at $r=R$. One earlier model has been considered by Wigner and Seitz [9], in which the potential arises from other atoms or molecules in a surrounding spherical lattice. ( see also the calculations by Aquino [10]).

Our model of confinement, using the flux $\mathrm{J}$, is a natural generalisation of earlier models and can be applied to an arbitrary surface enclosing the atom or molecule considered. A special case of our model is when the wavefunction is zero on a spherical surface and consequently $\mathrm{J}$ is zero. To achieve this confinement the applied field must be spherically symmetric and infinite on the surface. Using our generalisations both of these restrictions may be removed and we may apply the theory to model a wider set of physically confining fields. Changing the shape of the field enables us to treat systems that approach one-dimensional models and our theory also encompasses as a special case the models of atoms embedded in a lattice with different kinds of fields $[9,10]$ where the derivative of the wavefunction is zero. We make the same assumptions as the usual models at the bounding surface, namely that it is the effect of the outer potential that is modelled and we simply require $J=0$ on the surface in order to confine the system. In the following section, we present analogous results for H-like atoms for the special case of ellipsoidal confining surfaces.

\section{Theory and Calculations}

\subsection{A Simple Example}

Here we consider an ellipsoid so that the the vector describing points on the surface, $\mathbf{r}$, is defined by

$$
\mathbf{r}=(a \sin (\theta) \cos (\phi), a \sin (\theta) \sin (\phi), b \cos (\theta))^{T}
$$


where $a, b>0,0 \leq \theta \leq \pi, 0 \leq \phi \leq 2 \pi$. We construct

$$
h_{\theta} \mathbf{e}_{\theta}=\frac{\partial \mathbf{r}}{\partial \theta}=(\operatorname{acos}(\theta) \cos (\phi), \operatorname{acos}(\theta) \sin (\phi),-b \sin (\theta))^{T}
$$

where $\mathbf{e}_{\theta}$ is a unit vector and

$$
h_{\theta}=\sqrt{a^{2} \cos (\theta)^{2}+b^{2} \sin (\theta)^{2}}
$$

Similarly

$$
h_{\phi} \mathbf{e}_{\phi}=\frac{\partial \mathbf{r}}{\partial \phi}=(-\operatorname{asin}(\theta) \sin (\phi), \operatorname{asin}(\theta) \cos (\phi), 0)^{T}
$$

with

$$
h_{\phi}=\operatorname{asin}(\theta)
$$

The unit vectors obtained may be used to construct the normal to the surface

$$
\mathbf{n}=\frac{\mathbf{e}_{\theta} \times \mathbf{e}_{\phi}}{\left|\mathbf{e}_{\theta} \times \mathbf{e}_{\phi}\right|}=\frac{1}{h_{\theta}}(b \sin (\theta) \cos (\phi), b \sin (\theta) \sin (\phi), a \cos (\theta))^{T}
$$

With these definitions we may calculate J using

$$
J=\int_{0}^{2 \pi} \int_{0}^{\pi} \nabla \rho \cdot \mathbf{n} h_{\theta} h_{\phi} d \theta d \phi
$$

A simple example is that of a hydrogen atom in a S-state, situated at the centre of the ellipsoid. Since the potential is unchanged within the ellipsoid the form of the solution is unchanged and may be written in terms of the KummerM function:

$$
\psi=\exp (-\alpha r) M\left(1-\frac{1}{\alpha}, 2,2 \alpha r\right), \quad E=-\frac{1}{2} \alpha^{2}
$$

The difference between this function and the unconfined solution is simply that the energy has been changed by the confinement, so that it is parameterised by $\alpha$ which may be chosen to describe the confinement. Here we have

$$
\nabla \rho . \mathbf{n}=2 \psi \frac{\partial \psi}{\partial r} \hat{\mathbf{r}} . \mathbf{n}
$$

so that now $\mathrm{J}$ is a function of $\alpha$ and is given by

$$
J(\alpha)=4 \pi \int_{0}^{\pi} \psi \frac{\partial \psi}{\partial r} \frac{a^{2} b \sin (\theta)}{r} d \theta
$$

with

$$
r=\sqrt{a^{2} \sin (\theta)^{2}+b^{2} \cos (\theta)^{2}}, \quad h_{\theta} h_{\phi} \hat{\mathbf{r}} \cdot \mathbf{n}=\frac{a^{2} b \sin (\theta)}{r}
$$

Note that the wave function is independent of $\phi$ so that the integration over $\phi$ has been carried out analytically. 
In the case $a=b=2$ we have spherical confinement in a sphere of radius 2 . The lowest energy state is obtained where $J(1.2880269)=0$ and reproduces the result given by Aquino [10] and is the special case where the derivative of the wavefunction vanishes. For the second state we obtain $\alpha=0.5$ so that $J(0.5)=0$ and recover the solution given by $\mathrm{Eq}(4)$. This is a consequence of the fact that $\psi=0$ and the charge density is identically zero on the surface. It is important to note that even though the surface is the same for these solutions they represent different models with different confining fields and the corresponding wavefunctions are both nodeless. Similarly for the case $a=b=4$ we also have $J(0.5)=0$ and we also recover the solution in $\mathrm{Eq}(4)$, but this now corresponds to the derivative of the wavefunction being identically zero on the surface.Note that for a non-spherical bounding surface, zeroing the flux on the surface does not necessarily imply that the wavefunction or its normal derivative is zero everywhere on the surface. In tables 1-4 we give the negative energies of the confined states of H-like atoms with unit nuclear charge for specified values of $\mathrm{a}$ and $\mathrm{b}$ obtained by zeroing the flux over the surface.

Table 1: Confined Energies for various $a$ and increasing $b$

\begin{tabular}{c|c|c|c|c|c}
\hline \hline $\mathrm{a}$ & $\mathrm{b}$ & $\mathrm{E}$ & $\mathrm{a}$ & $\mathrm{b}$ & $\mathrm{E}$ \\
\hline 2 & 2 & -0.8295067 & 4 & 4 & -0.5293025 \\
2 & 2 & -0.1250000 & 4 & 4 & -0.4832653 \\
2 & 4 & -0.6647247 & 4 & 4 & -0.1250000 \\
2 & 4 & -0.2845238 & 4 & 10 & -0.5008145 \\
2 & 10 & -0.5068963 & 4 & 10 & -0.4992010 \\
2 & 10 & -0.4922178 & 4 & 10 & -0.1587450 \\
\hline \hline
\end{tabular}

Table 2: Confined Energies for $a=8$ with increasing and decreasing $b$

\begin{tabular}{c|c|c|c|c|c}
\hline \hline $\mathrm{a}$ & $\mathrm{b}$ & $\mathrm{E}$ & $\mathrm{a}$ & $\mathrm{b}$ & $\mathrm{E}$ \\
\hline 8 & 8 & -0.5000342 & 8 & 4 & -0.5007537 \\
8 & 8 & -0.4999751 & 8 & 4 & -0.4993037 \\
8 & 8 & -0.1581695 & 8 & 4 & -0.1662021 \\
8 & 8 & -0.0847387 & 8 & 2 & -0.5048701 \\
8 & 10 & -0.5000102 & 8 & 2 & -0.4949137 \\
8 & 10 & -0.4999915 & 8 & 2 & -0.1145893 \\
8 & 10 & -0.1512878 & 8 & 2 & -0.0525061 \\
8 & 10 & -0.0958895 & & & \\
\hline \hline
\end{tabular}

In table 2 the 4 negative energies for $a=b=8$ ( spherical confinement) and $a=8, b=2$ are fairly close and for $a=8, b=0$ these S-state energies will be identical with the spherical confinement. For the intermediate case of $a=8, b=4$ the spectrum is different and there are only 3 negative energies. 
These calculations can be extended in two ways. We can consider other states and in Table 3 below we give some results using $\mathrm{P}$ symmetric functions and this is achieved simply by changing the form of the wave function in Eq. (13) to

$$
\exp (-2 \alpha r) r \cos (\theta) M\left(2-\frac{1}{\alpha}, 4,2 \alpha r\right)
$$

We can also extend the calculations by changing the position of the atom within the ellipsoid.Here we fix the atom at the origin of the coordinates but change the centre of the ellipsoid so that the points are described by

$$
\mathbf{r}=\left(\operatorname{asin}(\theta) \cos (\phi)+x_{0}, a \sin (\theta) \sin (\phi)+y_{0}, b \cos (\theta)+z_{0}\right)^{T}
$$

In Table 4 we compare the results for $a=5, b=10$ in the case where the atom is at the centre of the ellipsoid with the results where the origin, and hence the atom is at the focus of the ellipsoid so that $x_{0}=y_{0}=0, z_{0}=\sqrt{b^{2}-a^{2}}$ for $b>a$.

Table 3: Confined P-State energies for various $a, b$

\begin{tabular}{c|c|c|c|c|c}
\hline \hline $\mathrm{a}$ & $\mathrm{b}$ & $\mathrm{E}$ & $\mathrm{a}$ & $\mathrm{b}$ & $\mathrm{E}$ \\
\hline 8 & 8 & -0.1489661 & 4 & 8 & -0.1657721 \\
8 & 8 & -0.1044501 & 4 & 8 & -0.0485718 \\
8 & 8 & -0.0096516 & 2 & 8 & -0.1712541 \\
8 & 12 & -0.1327892 & & & \\
8 & 12 & -0.1192630 & & & \\
8 & 12 & -0.0552281 & & & \\
\hline \hline
\end{tabular}

Table 4: Confinement with the atom displaced

\begin{tabular}{c|c|c|c}
\hline \hline $\mathrm{a}$ & $\mathrm{b}$ & Origin & Focus \\
\hline 5 & 10 & -0.5002774 & -0.5000087 \\
5 & 10 & -0.4997379 & -0.4999913 \\
5 & 10 & -0.1655912 & -0.1401402 \\
\hline \hline
\end{tabular}




\subsection{Confinement of valence orbitals}

The above treatment can be extended to larger atomic systems by approximating Schrödinger's equation for the outermost electron:

$$
-\frac{1}{2} \nabla^{2} \psi+V \psi=E \psi
$$

in a region $r>R_{a}$ for which

$$
V \approx-\frac{1}{r}
$$

We assume that the region $r \leq R_{a}$, which we describe as the core region, is not affected by the mechanism of confinement . For unconfined systems $E$ is taken to be the ionisation energy of the atom and since the core is not affected by the confinement we may take this value universally.

We consider only confining surfaces far from the atom so that the form of the wave function may be taken to be a solution of Eq. (19) where the potential is given by Eq. (20). To solve Eq. (19) outside the core we may take $\psi$ in the form

$$
\psi=k \exp (-\alpha r) r^{l} Y_{l m}(\theta, \phi) f(r), \quad E=-\frac{1}{2} \alpha^{2}, \quad \alpha>0
$$

where $Y_{l m}(\theta, \phi)$ is a spherical harmonic function. This leads to a Kummer equation for $f(r)$ with two independent solutions. The general solution may be written as

$$
f(r)=c M\left(l+1-\frac{1}{\alpha}, 2(l+1), 2 \alpha r\right)+U\left(l+1-\frac{1}{\alpha}, 2(l+1), 2 \alpha r\right)
$$

In the case of unconfined systems we require $\psi$ to vanish as $r \rightarrow \infty$ and since the Kummer M function is unbounded we need to choose $c=0$. This corresponds to the usual model of an isolated atom where no other atoms, molecules or fields are considered and the atom is treated as an independent entity. A modification of this model which still treats the atom as an independent entity would be to confine within a surface but of course since we are using the asymptotic form of the wavefunction the confining surface must be in a region where $r>R_{a}$ for consistency. If we consider such a confined system then we need to choose c so that the flux is zero on the surface.

Writing

$$
\psi_{1}=\exp (-\alpha r) r^{l} M\left(l+1-\frac{1}{\alpha}, 2(l+1), 2 \alpha r\right) Y_{l m}(\theta, \phi)
$$

and

$$
\psi_{2}=\exp (-\alpha r) r^{l} U\left(l+1-\frac{1}{\alpha}, 2(l+1), 2 \alpha r\right) Y_{l m}(\theta, \phi)
$$

we have

$$
J=2 \int_{S} \frac{\partial\left(c \psi_{1}+\psi_{2}\right)}{\partial n}\left(c \psi_{1}+\psi_{2}\right) d S
$$


If the surface is a sphere $r=R_{b}$ then terms in $\mathrm{r}$ may be taken out of the integral and the flux is zero at the two values of c where

$$
\left(c \psi_{1}+\psi_{2}\right)=0 \quad r=R_{b}
$$

or

$$
\frac{\partial\left(c \psi_{1}+\psi_{2}\right)}{\partial r}=0 \quad r=R_{b}
$$

leading to two different states degenerate in energy. For a more general surface the expression for the flux in Eq. (25) may be written in the form

$$
J=c^{2} J_{0}+c\left(J_{1}+J_{2}\right)+J_{3}
$$

where

$$
\begin{gathered}
J_{0}=2 \int_{S} \frac{\partial \psi_{1}}{\partial n} \psi_{1} d S, \quad J_{1}=2 \int_{S} \frac{\partial \psi_{1}}{\partial n} \psi_{2} d S \\
J_{2}=2 \int_{S} \frac{\partial \psi_{2}}{\partial n} \psi_{1} d S
\end{gathered}
$$

and

$$
J_{3}=2 \int_{S} \frac{\partial \psi_{2}}{\partial n} \psi_{2} d S
$$

If $l+1-1 / \alpha$ is not a non-positive integer, the Kummer M function is asymptotic to $\exp (2 \alpha r)$ as $r \rightarrow \infty$ so that, for sufficiently large $R_{a}$, the charge density $\left(\psi_{1}\right)^{2}$ is increasing . A similar, asymptotic argument leads to $\left(\psi_{2}\right)^{2}$ decreasing with $r$. We may deduce that $J_{0}>0$ and $J_{3}<0$. (In the case of a sphere the outer normal is simply $\hat{\mathbf{r}}$ and more generally the outer normal is in a direction where $r$ increases.)

Thus by choosing c sufficiently large and positive $J>0$ and similarly by choosing c sufficiently small $J<0$. It therefore follows that there are two real values of $c$ for which $J=0$ for all bounded surfaces sufficiently far from the atomic centre. In table 5 we consider Li with $l=0$ ( the ionisation energy is taken to be $E=-1.98174 a . u$. ) and the values of c are calculated for various values of the parameters a, b of an ellipsoid described in section 2. For the fixed value $a=5$ we see that the two values of c obtained both approach zero as b increases. In the final entry in the table we see that for a large spherical surface the two values of $c$ are very small and we essentially obtain the usual KummerU function for the asymptotic behaviour at infinity.

\subsection{Two-centre problems}

Here we consider examples of diatomic molecules and ions for example $\mathrm{H}_{2}^{+}$and $\mathrm{HeH}^{++}$.Previously we have treated such systems confined within an ellipsoidal region of the same shape as unconfined molecule [8] ( See also Cruz et al who treat these systems [7]).. Here we examine confinement inside a sphere of radius $r_{0}$ measured from an origin at the mid point of the interatomic line. The wave functions for the molecular systems are conveniently expressed in spheroidal 
Table 5: Values of $\mathrm{c}$ for given $\mathrm{a}$ and $\mathrm{b}$

\begin{tabular}{c|c|c|c}
\hline \hline $\mathrm{a}$ & $\mathrm{b}$ & $\mathrm{c}$ & $\mathrm{c}$ \\
\hline 5 & 5 & 0.71581618 & -5.60751045 \\
5 & 6 & 0.61441154 & -5.01990314 \\
5 & 10 & 0.38745042 & -1.16458622 \\
5 & 15 & 0.11314330 & -0.14144500 \\
5 & 20 & 0.01458050 & -0.01496838 \\
20 & 20 & 0.00000096 & -0.00000117 \\
\hline \hline
\end{tabular}

coordinates $p, q, \phi[11]$, and the points on the sphere may be written in terms of these coordinates in the form

$$
\begin{gathered}
\mathbf{r}=\frac{R}{2}\left(\sqrt{p^{2}-1} \sqrt{1-q^{2}} \cos (\phi), \sqrt{p^{2}-1} \sqrt{1-q^{2}} \sin (\phi), p q\right)^{T} \\
1 \leq p<\infty, \quad-1 \leq q \leq 1, \quad 0 \leq \phi \leq 2 \pi
\end{gathered}
$$

We can also express these points in terms of spherical coordinates:

$$
\mathbf{r}=r_{0}(\sin (\theta) \cos (\phi), \sin (\theta) \sin (\phi), \cos (\theta))^{T}
$$

which are appropriate for integration on the spherical surface. Thus we need to identify the connection between the two sets of coordinates on the surface. We have

which implies that

$$
r_{0}=\frac{R}{2} \sqrt{p^{2}+q^{2}-1}
$$

$$
p=+\sqrt{\omega-q^{2}}, \quad \omega=1+\frac{4 r_{0}^{2}}{R^{2}}
$$

Additionally

$$
r_{0} \cos (\theta)=\frac{R}{2} p q, \quad 0 \leq \theta \leq \pi
$$

so that for any $\theta$ both $p$ and $q$ are determined.The outward normal for the spherical surface is $\mathbf{n}=\mathbf{r} / r_{0}$, so that the required surface integral takes the form

$$
J=4 \pi \int_{0}^{\pi} \psi \nabla \psi \cdot \mathbf{n} \sin (\theta) d \theta
$$

where, as before, the integration over $\phi$ has been carried out analytically using the azimuthal symmetry. The approximate wave function $\psi$ is expressed in the form $X(p) Y(q)$ and

$$
\nabla \psi=\frac{1}{h_{p}} \frac{\partial \psi}{\partial p} \mathbf{e}_{\mathbf{p}}+\frac{1}{h_{q}} \frac{\partial \psi}{\partial q} \mathbf{e}_{\mathbf{q}}
$$

where

$$
\mathbf{e}_{\mathbf{p}}=\frac{1}{\sqrt{p^{2}-q^{2}}}\left(p \sqrt{1-q^{2}} \cos (\phi), p \sqrt{1-q^{2}} \cos (\phi), q \sqrt{p^{2}-1}\right)^{T}
$$




$$
\mathbf{e}_{\mathbf{q}}=\frac{1}{\sqrt{p^{2}-q^{2}}}\left(-q \sqrt{p^{2}-1} \cos (\phi),-q \sqrt{p^{2}-1} \cos (\phi), p \sqrt{1-q^{2}}\right)^{T}
$$

and

$$
h_{p}=\frac{R}{2} \sqrt{\frac{p^{2}-q^{2}}{p^{2}-1}}, \quad h_{q}=\frac{R}{2} \sqrt{\frac{p^{2}-q^{2}}{1-q^{2}}}
$$

The form of the approximate wave function $\psi(p, q)=X(p) Y(q)$ is discussed in the appendix ( and more details are given in [8] and [11]). We have carried out calculations for $\mathrm{H}_{2}^{+}$and $\mathrm{HeH}^{++}$in examples where $\mathrm{R}=r_{0}$. For the lowest states considered $X(p)$ asymptotically decays for large radial distances. Thus $X(p)$ will have an asymptotic factor $\exp (-\alpha r)$ where $\alpha$ is real and positive. This is not strictly necessary when the system is confined other possibilities correspond to very strong confinement leading to positive energies and purely imaginary values of $\alpha[4]$. The results are presented in tables 6 and 7 where the columns labelled $E_{+}$denote that $Y(q)$ is nodeless whereas the column labelled $E_{-}$denotes a single node in $Y(q)$. For $H_{2}^{+}$in table 6 the lower states have two kinds of approximate degeneracy. The symmetry under interchange of the two atoms leads to a classification of states in symmetric and antisymmetric states with respect to the intermolecular plane corresponding to the states $E_{+}$ and $E_{-}$respectively. As the internuclear distance, $R$, increases the two atoms approach independent systems and for all states

$$
E_{+} \rightarrow E_{-}
$$

For finite $R$ this will correspond to approximate degeneracy and for the values considered only the lower states that decay more rapidly, show this property. There is a second kind of approximate degeneracy for the lower states in each column for large $r_{0}$. For sufficiently large $r_{0}$ compared with $R$ the system approaches a spherical confinement where we can choose either the wave function or the normal derivative zero on the spherical surface. For any radial confinement the normal derivative is $\partial \psi / \partial r$ and if both this derivative and $\psi$ are zero on the sphere, then from the Schrodinger equation we can deduce the the secondorder radial derivative is zero. Further radial differentiation of the Schrodinger equation shows that this implies all radial derivatives will be zero on the surface. A simple Taylor expansion along any radial path toward the origin then shows that $\psi=0$ within the sphere. Thus the wavefunction and its derivative cannot both be zero on a sphere with finite radius. But for $r_{0} \rightarrow \infty$ the exponential decay ensures that both the wave function and derivative approach zero and the energy obtained from zeroing both the wave function and derivative becomes the same. For large but finite $r_{0}$ this leads to the approximate degeneracy shown in table 6 for the lower states and for larger confining radii all asymptotic decaying states will be paired in this way. The results for $\mathrm{HeH}^{++}$are presented in the same way in table 7 but here only the second approximate degeneracy is apparent since the system is not symmetric in the internuclear plane. The lowest energies are negative but eventually positive energies occur especially for 
the lower values of $R$. The energy expression for the two-centre problem is

$$
E=-2 \alpha^{2}+\frac{Z}{R}
$$

where $Z=1$ for $\mathrm{H}_{2}^{+}$and $Z=2$ for $\mathrm{HeH}^{++}$. Thus the positive energies can occur in two ways: when $\alpha$ is real but the factor in $R^{-1}$ is such that the total energy is positive or when $\alpha$ is purely imaginary. There are only two values in tables 6 and 7 where the latter is the case and they are presented in italics.

Table 6: Lowest states for $\mathrm{H}_{2}^{+}$

\begin{tabular}{c|c|c}
\hline \hline$R=r_{0}$ & $E_{+}$ & $E_{-}$ \\
\hline 8 & -0.503724 & -0.499805 \\
8 & -0.500832 & -0.496676 \\
8 & -0.108465 & -0.082560 \\
8 & 0.016197 & 0.063495 \\
5 & -0.543718 & -0.499626 \\
5 & -0.503844 & -0.448622 \\
5 & -0.065420 & 0.074483 \\
2 & -0.976539 & -0.482567 \\
2 & -0.181572 & 1.250653 \\
\hline \hline
\end{tabular}

Table 7: Lowest states for $\mathrm{HeH}^{++}$

\begin{tabular}{c|c|c}
\hline \hline$R=r_{0}$ & $E_{+}$ & $E_{-}$ \\
\hline 8 & -1.875040 & -0.504093 \\
8 & -1.875028 & -0.501773 \\
8 & -0.371120 & -0.139337 \\
8 & -0.313179 & -0.029149 \\
8 & 0.0773572 & 0.139461 \\
5 & -1.801448 & -0.538861 \\
5 & -1.798839 & -0.506830 \\
5 & -0.315201 & -0.073437 \\
5 & 0.042203 & 0.324245 \\
2 & -1.796769 & -0.730351 \\
2 & -0.125214 & 0.180375 \\
2 & 0.778784 & 2.021185 \\
\hline \hline
\end{tabular}




\section{Conclusion}

In this paper we have reconsidered the problem of confinement of atomic and molecular systems from first principles. In practice no system is isolated and the models used should reflect that there is a field acting upon the atomic or molecular systems. The usual models of confinement do not specify the field accurately and most models simply describe the effect of the confining field on some boundary. We make the same assumption but choose the zero of the outward flux on some general surface to be zero thereby confining the system within the surface. In the case of one-centre problems with spherical symmetry we present examples where the confining surface was elliptical and for two-centre problems, where the symmetry of the system is elliptical we use a spherical confining surface. The change of symmetry naturally involves more sophisticated calculations than simply choosing the wave function or its derivative zero on a surface with the same symmetry, but the examples illustrate that the theory can be applied for any closed surface enclosing the atomic or molecular system where a single valence orbital describes the asymptotic properties. However it may be possible to treat a set of parameterised valence orbitals, within the one electron approximation, but this will be a more difficult problem numerically.

\section{APPENDIX}

For the two-centred problems, with azimuthal symmetry we may separate the Schrödinger equation into a pair of equations, coupled through a constant $\mathrm{C}$ to be determined, in the independent variables $p$ and $q$ :

$$
\begin{gathered}
\frac{d}{d p}\left\{\left(p^{2}-1\right) \frac{d X}{d p}\right\}+\left\{-C-\lambda^{2}\left(p^{2}-1\right)+R\left(\mu_{a}+\mu_{b}\right) p\right\} X=0 \\
\frac{d}{d q}\left\{\left(1-q^{2}\right) \frac{d Y}{d q}\right\}+\left\{C-\lambda^{2}\left(1-q^{2}\right)-R\left(\mu_{a}-\mu_{b}\right) q\right\} Y=0
\end{gathered}
$$

where $\lambda=R \alpha, \mu_{a}, \mu_{b}$ are the charges on the two centres A and B. Essentially we may write

$$
X=\exp \left(-\frac{\alpha x}{2}\right) \sum_{n=0}^{\infty} c_{n} x^{n}
$$

where $x=2 R(p-1)$. This ensures that for lower states and large $\mathrm{R}$ we have the same form of asymptotic behaviour as for the unconfined systems, but for higher states with positive energies, $\alpha$ may be purely imaginary. The coefficients $c_{n}$ satisfy the recurrence relation

$$
\begin{gathered}
(n+1)^{2} c_{n+1}=\left\{\frac{1}{2}(2 n+1) \alpha-\frac{n(n+1)}{4 R}+\frac{C}{4 R}-\frac{t}{R}\right\} c_{n} \\
+\left\{\frac{\alpha n}{4 R}-\frac{t}{8 R}\right\} c_{n-1}
\end{gathered}
$$


where $t=\mu_{a}+\mu_{b}, c_{0}=1, c_{-1}=0$. The separation constant $C=\lambda^{2}-\epsilon_{q}$ is determined by solving Eq 41 approximately using a matrix eigenvalue problem. To do this we expand $Y$ in the form

$$
Y=\sum_{n=0}^{N} a_{n} P_{n}(q)
$$

where $P_{n}(q)$ is the Legendre polynomial of degree and $\epsilon_{q}$ is an eigenvalue of the constructed matrix. This eigenvalue can be chosen according to the number of nodes in Y. Full details of the matrix elements are given in [8] .

Given the wave function $\psi=X(p) Y(q)$ the integrations were carried out using a simple application of Newton's method and were completed in MAPLE. The truncation of the series for $\mathrm{X}$, the order of the matrix for $\mathrm{Y}$ and the number of points in the integration process can all be adjusted to achieve a desired accuracy, but in practice, the accuracy of the model will be determined by the effect of the confining field.

[ 1 ] Advances in Quantum Chemistry, Edited by J.R. Sabin, E.Brandas and S.Cruz, Vol 57 (Academic Prees,London,2009).

[ 2 ] H.E. Montgomery Jr and K.D. Sen, Physics Lett. A 376, 1992 (2012).

[ 3 ] H.E.Montgomery Jr, Euro. J.Phys. 32 , 1275 (2011).

[ 4 ]B.L. Burrows and M.Cohen, Int.J. Quant. Chem. 106, 478 (2006).

[ 5 ] B.L. Burrows and M. Cohen, Phys. Rev. A 72, 032508 (2005).

[ 6 ] C. Laughlin and S.I-Chu, J.Phys.A:Math.Theor. 42, 265004 (2009).

[ 7 ] S.A.Cruz and R. Colin-Rodriguez,Int. J. Quant.Chem. 109,3041 (2009).

[ 8 ] B.L. Burrows and M. Cohen, Phys. Rev. A 88, 052511 (2013).

[ 9 ] E. Wigner, F.Seitz, Phys. Rev. 43804 (1933).

[ 10 ] N.Aquino,Advances in Quantum Chemistry, Vol 57 ,123 (2009).

[ 11 ] B.L. Burrows , A.Dalgarno and M. Cohen, Phys. Rev.A 81, 042508 (2010). 